Histone epiproteomic profiling distinguishes oligodendroglioma, IDH-mutant and $1 \mathrm{p} / 19 \mathrm{q}$ co-deleted from IDH-mutant astrocytoma and reveals less tri-methylation of $\mathrm{H} 3 \mathrm{~K} 27$ in oligodendrogliomas

Feller, C ; Felix, M ; Weiss, T ; Herold-Mende, C ; Zhang, F ; Kockmann, T ; Sahm, F ; Aebersold, R ; von Deimling, A ; Reuss, D E

DOI: https://doi.org/10.1007/s00401-019-02096-8

Posted at the Zurich Open Repository and Archive, University of Zurich

ZORA URL: https://doi.org/10.5167/uzh-177688

Journal Article

Accepted Version

Originally published at:

Feller, C; Felix, M; Weiss, T; Herold-Mende, C; Zhang, F; Kockmann, T; Sahm, F; Aebersold, R; von Deimling, A; Reuss, D E (2020). Histone epiproteomic profiling distinguishes oligodendroglioma, IDHmutant and 1p/19q co-deleted from IDH-mutant astrocytoma and reveals less tri-methylation of H3K27 in oligodendrogliomas. Acta Neuropathologica, 139(1):211-213.

DOI: https://doi.org/10.1007/s00401-019-02096-8 
Histone epi-proteomic profiling distinguishes Oligodendroglioma, IDH-mutant and 1p/19q co-deleted from IDH-mutant Astrocytoma and reveals less tri-methylation of H3K27 in Oligodendrogliomas

C. Feller ${ }^{1}$, M. Felix ${ }^{2}$, T. Weiss ${ }^{3}$, C. Herold-Mende ${ }^{4}$, F. Zhang ${ }^{1}$, T. Kockmann 5 , F.Sahm², , R. Aebersold ${ }^{1,7}$, A. von Deimling ${ }^{2,6}$ and D.E. Reuss ${ }^{2,6}$

1 Department of Biology, Institute of Molecular Systems Biology, ETH Zürich, Otto Stern Weg 3, 8093, Zürich, Switzerland 2 Clinical Cooperation Unit Neuropathology, German Consortium for Translational Cancer Research (DKTK), German Cancer Research Center (DKFZ), Heidelberg, Germany.

3 Department of Neurology and Brain Tumor Center, University Hospital Zurich and University of Zurich, 8091 Zurich, Switzerland.

4 Division of Experimental Neurosurgery, Department of Neurosurgery, University Hospital Heidelberg, Heidelberg, Germany.

5 Functional Genomics Center Zurich, Swiss Federal Institute of Technology Zurich, University of Zurich, Winterthurerstr. 190, CH-8057 Zurich, Switzerland.

6 Department of Neuropathology, Institute of Pathology, University Hospital Heidelberg, Heidelberg, Germany.

7 Faculty of Science, University of Zürich, Zürich, Switzerland

Corresponding authors:

Christian Feller, $\mathrm{PhD}$

Department of Biology,

Institute of Molecular Systems Biology,

ETH Zürich, Otto Stern Weg 3, 8093, Zürich, Switzerland

fellerc@ethz.ch

and

David E. Reuss

Department of Neuropathology

University Hospital Heidelberg

and

Clinical Cooperation Unit Neuropathology (B300)

German Cancer Research Center (DKFZ)

Im Neuenheimer Feld 224

69120 Heidelberg, Germany

david.reuss@med.uni-heidelberg.de 
WHO classification for tumors of the central nervous system strongly endorses molecular tests for the precise diagnosis of diffuse gliomas. Subclassification of isocitrate dehydrogenase (IDH)-mutant gliomas into IDH-mutant astrocytomas and oligodendrogliomas requires testing for complete $1 \mathrm{p} / 19 \mathrm{q}$ co-deletion [1] in those cases not showing loss of ATRX expression [2].

Epigenetics recently emerged as a promising new avenue for the classification of gliomas and driver mutations in epigenetic regulators like IDH1/2 and $\mathrm{H} 3 \mathrm{~F} 3 \mathrm{~A}$ are present in gliomas and are characteristic for distinct subtypes [3]. Comparison of methylation profiles generated by chip analysis has been shown to be a powerful tool to distinguish between brain tumor entities [4]. In addition to DNA methylation, post-translational modifications (PTMs) at histone proteins form the basis of epigenetic regulation.

Despite progress into classification substantial limitations for diagnosis remain, e.g. valid $1 \mathrm{p} / 19 \mathrm{q}$ testing requires array technologies or next-generation-sequencing approaches. Furthermore, while alterations in the DNA methylation status of gliomas are already well documented, changes to the epigenetic layer at the level of histone modifications are only poorly characterised.

The role of $\mathrm{H} 3 \mathrm{~K} 27$ tri-methylation in several brain tumor entities is well established [58]. We assessed H3K27me3 by immunohistochemistry in a series of 118 IDH-mutant oligodendrogliomas and astrocytomas classified by DNA methylation profiling including 1p/19q status determination (Figure 1a, Supplementary table 1). We used two of the most widely applied antibodies, which consistently showed tumor cell-specific reduction in DMGH3K27M, EPN_PFA and PRC2 inactivated MPNST [5-8]. We found that these two H3K27me3 antibodies generated strikingly different staining patterns: while the polyclonal antibody (07-449, Millipore) shows retained H3K27me3 in all samples analysed (10/10 oligodendrogliomas and 10/10 astrocytomas), the monoclonal C36B11 antibody shows a complete loss of nuclear staining in almost all oligodendrogliomas (loss in 74/76, 97\%) while astrocytomas showed retained expression in the vast majority of cases (loss in 7/42, 17\%). Using clone C36B11 similar results were recently reported in an independent study [9].

In order to get an antibody-independent and more quantitative insight we applied a recently developed data-independent acquisition (DIA) - mass spectrometry method to determine the H3K27 methylation status. The H3K27me3-levels of 12 measured oligodendroglioma samples were on average 2-fold lower compared with 14 astrocytoma samples (Figure 1B). While oligodendrogliomas showed a mean fraction of $4.6 \% \mathrm{H} 3 \mathrm{~K} 27 \mathrm{me} 3$, 
the astroglioma samples had $10.4 \%$ of H3K27me3. A similar 2-fold difference was found for the H3.3K27me3 mark at the histone isoform H3.3 (H3.3K27me3: $2.7 \%$ vs $6 \%$ ). Of note, to ensure high specificity, all histone mass spectrometric measurements were established and calibrated with synthetic reference peptides harboring the modification of interest. In summary, highly specific mass spectrometry measurements confirmed the lower levels of H3K27me3 in oligodendrogliomas.

In addition to single methylation sites, mass spectrometry-based quantification of protein modifications also allows determination of complex combinatorial modification patterns, aka histone modification motifs $[10,11]$. From the 38 differently modified histone motifs encompassing all possible combinations of mono-, di- and tri-methylation on H3K27 and $\mathrm{H} 3 \mathrm{~K} 36$, as well as acetylation on $\mathrm{H} 3 \mathrm{~K} 27$, five modification motifs showed more than 2fold differences between the oligodendroglioma and astrocytoma samples (Supplementary Figure 1a).

We next generated a comprehensive histone epi-proteomic map that documents the abundance of almost all characterized and many uncharacterized histone modifications at the five major histone proteins (Figures 1c, Supplementary Figure 1b). The DIA - mass spectrometry protocol recorded all possible peptide signatures and allowed extraction of these signals after so called "targeted assays" had been established, a procedure that connects a mass spectrometry signal with the peptide of interest by using spectra from a library of synthetic reference peptides as ground truth. We generated a DIA library of over 300 histone peptides that contain 17 different modification types. Across the 26 samples, we consistently detected and quantified 144 histone peptides. Among them were lysine acetylations and methylations at 17 different histone sequence isoforms, phosphorylation and butyrylations at histone H3, propionylation at histone $\mathrm{H} 4$ and ubiquitylations at histones $\mathrm{H} 3$ and $\mathrm{H} 2 \mathrm{~A}$ (Supplementary Figure 1b, Supplementary table 2). 45 histone peptides were significantly different between the oligodendroglioma and astrocytoma samples $(\mathrm{p}<0.05$, FDR-adjusted $\mathrm{p}$-values after performing 2-sided unpaired t-test). In addition to $\mathrm{H} 3 / \mathrm{H} 3.3 \mathrm{~K} 27 \mathrm{me} 3$, another repressive mark (H3 $\mathrm{K} 9 \mathrm{me} 3 \mathrm{~K} 14 \mathrm{ac}$ ) was depleted in oligodendrogliomas. Oligodendrogliomas also showed an enrichment of acetylated lysine 9 at histone H2AX (H2AXK9ac) and ubiquitylated lysine 15 on H2AJ (H2AJ K13ac K15ub), two poorly-characterised histone modifications that have been linked to DNA damage repair $[12,13]$. Most of the oligodendroglioma samples also showed higher levels of butyrylated lysine 18 on $\mathrm{H} 3(\mathrm{H} 3 \mathrm{~K} 18 \mathrm{bu})$, which is a yet to be characterised histone modification type. 
An unbiased unsupervised clustering analysis using all 144 quantified peptides separated the two IDH-mutant glioma subtypes in full accordance to the EPIC DNA methylation classifier and the $1 \mathrm{p} / 19 \mathrm{q}$ status (Figure 1c and Supplementary Figure 1c). Restricting the clustering analysis to the 45 significantly different histone peptides further increased the correlations within the groups of oligodendrogliomas and astrocytomas. The clustering also revealed at least two histone epi-proteomic subgroups of oligodendroglioma, a feature not observable in the DNA methylation dataset (Figure 1c). These oligodendroglioma subgroups vary in a number of histone peptides, including the active marks H3K36me1 and H3K36me2. Of note, the separation of astrocytomas and oligodendrogliomas was less apparent when we used the 46 histone peptides that are commonly reported in histone mass spectrometry analysis (Supplementary Figures 1c). Furthermore, we noted that while most individual histone modifications show only moderate differences between the two glioma subtypes, the combination of multiple such histone motifs increases the discriminatory power to differentiate oligodendroglioma from astrocytoma.

In summary, so far a comprehensive analysis of the histone epi-proteome of brain tumors was lacking. By applying a DIA-based mass spectrometry method, our results indicate that histone epi-proteomic profiling at the depth of the current method has the capacity to identify clinically-relevant glioma sub-groups. Our analysis documented significant abundance differences in almost one-third of the 144 quantified histone peptides. This study therefore provides the first evidence for a substantial differential alteration of the histone epi-proteome in IDH-mutant glioma subtypes suggesting that $1 \mathrm{p} / 19 \mathrm{q}$ co-deletion status may interfere with epigenetic modifications as well. In addition to being of potential use for diagnostic purposes (supplementary note), this could also provide novel insights in glioma biology and may identify new therapeutic targets.

Acknowledgements For excellent technical support we sincerely thank V. Zeller, U. Vogel and J. Reuss. C. Feller acknowledges support by the DFG (FE-1544/1-1) and EMBO (long-term postdoctoral fellowship ALTF 55-2015). F. Sahm is a fellow of the Else Kröner Excellence Program of the Else Kröner-Fresenius Stiftung (EKFS). 


\section{Figure legend}

Fig. 1a: H3K27me3 immunohistochemistry. Antibody C36B11 (upper left) and antibody 07449 (bottom left) show retained expression in astrocytoma. Antibody C36B11 shows tumor cell specific lack of nuclear expression in oligodendroglioma (upper right) while antibody 07-449 shows retained expression in the same oligodendroglioma (bottom right). Scale bar $=200 \mu \mathrm{m}$ b: Oligodendrogliomas have significantly lower H3K27 tri-methylation (H3K27me3) than astrocytomas $(\mathrm{p}<0.05$, FDR-adjusted $\mathrm{p}$-values after performing 2 -sided unpaired $\mathrm{t}$-test). Box plot analysis comparing tri-methylation at lysine 27 between oligodendrogliomas and astrocytomas at the canonical histone H3.1/H3.2 (top panel) and the histone variant H3.3 (bottom panel). Values correspond to the estimated fraction of methylation on the ArgC-like peptide ranging from lysine 27 to arginine 40. c: Unsupervised hierarchical clustering analysis separates oligodendrogliomas from astrocytomas. Clustering was performed on a pairwise Spearman correlation matrix using the standard setting in the R package "heatmap.2", i.e. the "complete" method. Cutting the dendrogram at the second level generates two clusters of the oligodendroglioma dataset and one cluster in the astrocytoma dataset. Sample descriptions are shown above the heat map (sex, age, WHO status, histology, DNA methylome based on the EPIC classifier). 


\section{References}

1. Louis, D.N., et al., The 2016 World Health Organization Classification of Tumors of the Central Nervous System: a summary. Acta Neuropathol, 2016. 131(6): p. 803-20.

2. Reuss, D.E., et al., ATRX and IDH1-R132H immunohistochemistry with subsequent copy number analysis and IDH sequencing as a basis for an "integrated" diagnostic approach for adult astrocytoma, oligodendroglioma and glioblastoma. Acta Neuropathol, 2015. 129(1): p. 133-46.

3. Sturm, D., et al., Hotspot mutations in H3F3A and IDH1 define distinct epigenetic and biological subgroups of glioblastoma. Cancer Cell, 2012. 22(4): p. 425-37.

4. Capper, D., et al., DNA methylation-based classification of central nervous system tumours. Nature, 2018. 555(7697): p. 469-474.

5. Bender, S., et al., Reduced H3K27me3 and DNA hypomethylation are major drivers of gene expression in K27M mutant pediatric high-grade gliomas. Cancer Cell, 2013. 24(5): p. 660-72.

6. Prieto-Granada, C.N., et al., Loss of H3K27me3 Expression Is a Highly Sensitive Marker for Sporadic and Radiation-induced MPNST. Am J Surg Pathol, 2016. 40(4): p. 479-89.

7. Rohrich, M., et al., Methylation-based classification of benign and malignant peripheral nerve sheath tumors. Acta Neuropathol, 2016. 131(6): p. 877-87.

8. Venneti, S., et al., Evaluation of histone 3 lysine 27 trimethylation (H3K27me3) and enhancer of Zest 2 (EZH2) in pediatric glial and glioneuronal tumors shows decreased H3K27me3 in H3F3A K27M mutant glioblastomas. Brain Pathol, 2013. 23(5): p. 558-64.

9. Filipski, K., et al., Lack of H3K27 trimethylation is associated with $1 p / 19 q$ codeletion in diffuse gliomas. Acta Neuropathol, 2019. 138(2): p. 331-334.

10. Blasi, T., et al., Combinatorial Histone Acetylation Patterns Are Generated by Motif-Specific Reactions. Cell Syst, 2016. 2(1): p. 49-58.

11. Feller, C., et al., Global and specific responses of the histone acetylome to systematic perturbation. Mol Cell, 2015. 57(3): p. 559-71.

12. van Attikum, H. and S.M. Gasser, Crosstalk between histone modifications during the DNA damage response. Trends Cell Biol, 2009. 19(5): p. 207-17.

13. Zentner, G.E. and S. Henikoff, Regulation of nucleosome dynamics by histone modifications. Nat Struct Mol Biol, 2013. 20(3): p. 259-66. 